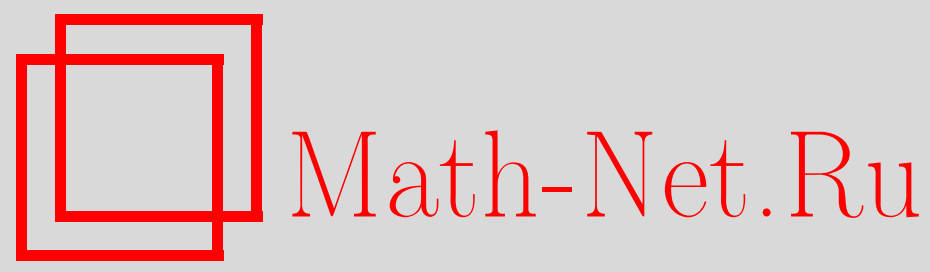

Т. Я. Азизов, Л. И. Сухочева, В. А. Штраус, Операторы в пространстве Крейна, Матем. заметки, 2004, том 76, выпуск $3,324-334$

DOI: https://doi.org/10.4213/mzm109

Использование Общероссийского математического портала Math-Net.Ru подразумевает, что вы прочитали и согласны с пользовательским соглашением http://www . mathnet.ru/rus/agreement

Параметры загрузки:

IP : 18.234 .197 .8

26 апреля 2023 г., $16: 17: 46$

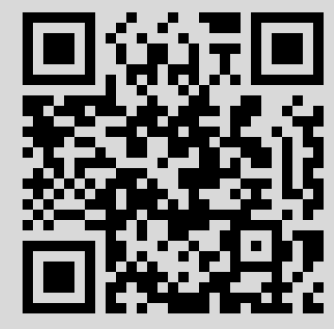




\title{
ОПЕРАТОРЫ В ПРОСТРАНСТВЕ КРЕЙНА
}

\author{
Т. Я. Азизов, Л. И. Сухочева, В.А. Штраус
}

\begin{abstract}
Изучаются самосопряженные операторы в пространстве Крейна. Цель работы показать связь между следующими классами операторов: операторы с компактньм "уголком", дефинизируемые операторы, операторы классов $(\mathbf{H})$ и $\mathbf{K}(\mathbf{H})$, и операторы класса $D_{\kappa}^{+}$.

Библиография: 10 названий.
\end{abstract}

1. Введение и основные понятия. Пусть $\mathscr{K}$ - линейное пространство, снабженное индефинитной метрикой (или, что то же, полутора-линейной формой) $[\cdot, \cdot]$. Предположим, что $\mathscr{K}$ допускает разложение

$$
\mathscr{K}=\mathscr{K}^{+}+\mathscr{K}^{-}
$$

в прямую ортогональную сумму положительного $\mathscr{K}^{+}$и отрищательного $\mathscr{K}^{-}$подпространств. Если $\left\{\mathscr{K}^{+},[\cdot, \cdot]\right\}-$ гильбертово, а $\left\{\mathscr{K}^{-},[\cdot, \cdot]\right\}-$ анти-гильбертово пространства (последнее означает, что $\left\{\mathscr{K}^{-},-[\cdot, \cdot]\right\}$ - гильбертово пространство), то $\mathscr{K}$ назьвают пространством Крейна.

Обозначим через $P^{+}$и $P^{-}$проекторы на $\mathscr{K}^{+}$и $\mathscr{K}^{-}$, соответствующие разложению (1.1), и введем оператор $J=P^{+}-P^{-}$. Тогда $\mathscr{K}$ будет гильбертовым относительно скалярного произведения $(\cdot, \cdot)=[J \cdot, \cdot]$. Отсюда, $[\cdot, \cdot]=(J \cdot, \cdot)$. Пространство Крейна с так введенным скалярньм произведением назьвают $J$-nространством, а индефинитную метрику - $J$-метрикой. Заметим, что разложение (1.1) ортогонально как относительно $J$-метрики, так и относительно гильбертова скалярного произведения.

Подпространство $\mathscr{L} \subset \mathscr{K}$ называется регулярным, если

$$
\mathscr{K}=\mathscr{L}[\dot{+}] \mathscr{L}^{[\perp]},
$$

где через $\mathscr{L}^{[\perp]}$ обозначено $J$-ортогональное дополнение к $\mathscr{L}$. В отличие от гильбертова случая для пространств с индефинитной метрикой подпространство $\mathscr{L}^{0}=\mathscr{L} \cap \mathscr{L}^{[\perp]}$, назьваемое изотропным, не обязательно тривиально, но даже если и тривиально, то

Работа выполнена при поддержке Российского фонда фундаментальных исследований, гранты № 02-01-00353, NWO-RFBR № 047-008-008, и проекта CONICIT (Венесуэла), грант № 97000668.

(С) Т. Я. Азизов, Л.И. СухочевА, В.А. ШтрАуС 2004 
равенство (1.2) может не иметь место. Отметим, что $\mathscr{L}$ регулярно тогда и только тогда, когда оно само является пространством Крейна. В частности, гильбертовы и анти-гильбертовы подпространства являются регулярными. Последние также назьваются равномерно положительными и равномерно отрицательными подпространствами, соответственно.

Говорят, что подпространство $\mathscr{L}_{+}\left(\mathscr{L}_{-}\right)$принадлежит $к л а с с у \mathbf{h}^{+}\left(\mathbf{h}^{-}\right)$, если $\mathscr{L}_{+}^{0}$ $\left(\mathscr{L}_{-}^{0}\right)$ конечномерно, а фактор-пространство $\widehat{\mathscr{L}}_{+}=\mathscr{L}_{+} / \mathscr{L}_{+}^{0}\left(\widehat{\mathscr{L}}_{-}=\mathscr{L}_{-} / \mathscr{L}_{-}^{0}\right)$ является гильбертовым (анти-гильбертовым) относительно индуцированной индефинитной метрики, или, что эквивалентно, $\mathscr{L}_{+} \in \mathbf{h}^{+}\left(\mathscr{L}_{-} \in \mathbf{h}^{-}\right)$, если оно разлагается в сумму конечномерного изотропного и равномерно положительного (равномерно отрицательного) подпространств.

Множество максимальных неотрицательных и максимальных неположительных подпространств будем обозначать через $\mathfrak{M}^{+}$и $\mathfrak{M}^{-}$, соответственно.

Пространство Крейна с $\kappa=\min \left\{\operatorname{dim} \mathscr{K}^{+}, \operatorname{dim} \mathscr{K}^{-}\right\}<\infty$ называют пространством Понтрягина $с к$ положительными или отрицательными квадратами, в зависимости от того будет ли $\kappa=\operatorname{dim} \mathscr{K}^{+}$или $\kappa=\operatorname{dim} \mathscr{K}^{-}$, соответственно.

Одной из основных проблем теории операторов в пространствах Крейна является проблема существования максимальных неотрицательных и максимальных неположительных инвариантных подпространств у $J$-самосопряженных и, эквивалентно, $J$-унитарных операторов. Или в более общем виде: проблема расширения заданного неотрицательного или неположительного инвариантного подпространства до максимального в своем классе инвариантного подпространства. И, наконец, эти же проблемы для семейств операторов. В достаточно полной мере эти задачи решены для случая пространства Понтрягина. В случае общего пространства Крейна ни одна из этих задач не решена, однако получен ряд достаточных условий, при которых они имеют решения (см., например, [1]-[3], и др.); к этим же источникам отсылаем читателя за подробностями геометрии и теории операторов пространств с индефинитной метрикой.

Мы выделяем 4 основных класса операторов:

(i) операторы с вполне непрерывным "уголком";

(ii) дефинизируемые операторы;

(iii) операторы класса $\mathbf{K}(\mathbf{H})$;

(iv) операторы класса $\mathbf{D}_{\kappa}^{+}$.

Сведения по классам (i), (ii) см., например, в [1]-[3], о классе $\mathbf{K}(\mathbf{H})$ и подклассе $\mathbf{H}-$ в [2] и [4], об операторах класса $\mathbf{D}_{\kappa}^{+}$см. [5], [6]. В дальнейшем изложении будем предполагать, если не оговорено другое, все операторы ограниченными и заданными на всем пространстве.

Цель настоящего исследования - провести сравнительньй анализ указанных классов операторов.

\section{2. Классы операторов. Определения.}

ОпРЕДЕЛЕНИЕ 2.1. Пусть $\mathscr{K}$ - пространство Крейна и пусть $T$ - непрерьвньй оператор и

$$
T=\left[\begin{array}{ll}
T_{11} & T_{12} \\
T_{21} & T_{22}
\end{array}\right]
$$

- его матричноепредставление относительно разложения (1.1). Будем говорить, что непрерьвньй оператор $T$ имеет вполне непрерывный уголок и писать $T \in \mathfrak{S}_{\infty, 12}$, если 
существует такое разложение (1.1), что $T_{12}$ - вполне непрерывный оператор $\left(T_{12} \in \mathfrak{S}_{\infty}\right)$.

ОпРЕДЕЛЕНИЕ 2.2. Самосопряженный оператор $A$, действующий в пространстве Крейна $\mathscr{K}$, назьвают дефинизируемым, если существует многочлен $p$ такой, что $p(A)-$ неотрицательный оператор, т.е. $[p(A) x, x] \geqslant 0$ при всех $x \in \mathscr{K}$. Множество дефинизируемых операторов обозначим символом $\mathbf{D}$.

ОПРЕДЕЛЕНИЕ 2.3. Будем говорить, что оператор Т принадлежит классу $\mathbf{H}$ и писать $T \in \mathbf{H}$, если у него существуют инвариантные подпространства $\mathscr{L}^{ \pm} \in \mathfrak{M}^{ \pm}$и все такие подпространства принадлежат классу $\mathbf{h}^{ \pm}$, соответственно.

ОПРЕДЕЛЕНИЕ 2.4. Множество $\mathfrak{A}=\{A\}$ самосопряженных линейных операторов, действующих в пространстве Крейна, принадлежит классу $\mathbf{K}(\mathbf{H}), \mathfrak{A} \in \mathbf{K}(\mathbf{H})$, если сушествует такой самосопряженньй оператор $B \in \mathbf{H}$ в этом пространстве, что $B A=A B$ при всех $A \in \mathfrak{A}$. Если $\mathfrak{A}$ состоит из одного оператора $A$, то будем говорить, что $A$ принадлежит классу $\mathbf{K}(\mathbf{H})$ и писать $A \in \mathbf{K}(\mathbf{H})$.

ОПРЕДЕЛЕниЕ 2.5. Скажем, что семейство $\mathfrak{A}=\{A\}$ самосопряженных линейных операторов, действующих в пространстве Крейна, принадлежсит классу $\mathbf{D}_{\kappa}^{+}, \mathfrak{A} \in \mathbf{D}_{\kappa}^{+}$, если для него найдется пара инвариантных подпространств $\mathscr{L}^{ \pm} \in \mathfrak{M}^{ \pm} \cap \mathbf{h}^{ \pm}, \mathscr{L}^{+}[\perp] \mathscr{L}^{-}$, с размерностью подпространства $\mathscr{L}^{+} \cap \mathscr{L}^{-}$, равной $\kappa, \kappa<\infty$.

Установим связь и различие этих классов операторов. Прежде всего заметим, что из [2, теорема 3.5.16] следует $\mathbf{K}(\mathbf{H}) \subset \mathbf{D}_{\kappa}^{+}$. Мы покажем (см. ниже теорему 4.1), что конечное семейство самосопряженных операторов принадлежит $\mathbf{D}_{\kappa}^{+}$тогда и только тогда, когда оно принадлежит классу $\mathbf{K}(\mathbf{H})$.

Пусть $\mathfrak{A} \in \mathbf{D}_{\kappa}^{+}$- коммутативное семейство самосопряженных операторов в пространстве Крейна. Из [2, теорема 3.5.2] вытекает существование такого разложения (1.1), что все операторы из $\mathfrak{A}$ имеют вполне непрерьвные уголки. В следующем разделе будут приведены примеры, показьвающие различие этих классов операторов.

3. Примеры. Ниже в примерах 3.1 и 3.2 предполагается, что

$$
\mathscr{H}=\mathscr{G} \oplus \mathscr{G}
$$

- ортогональная сумма двух экземпляров бесконечномерного гильбертова пространства $\mathscr{G}$, а $J$-метрика в $\mathscr{H}$ введена с помощью оператора

$$
J=\left[\begin{array}{ll}
0 & I \\
I & 0
\end{array}\right]
$$

ПримеР $3.1\left(\mathfrak{S}_{\infty, 12} \not \subset(\mathbf{D} \cup \mathbf{K}(\mathbf{H}))\right.$. Пусть $A: \mathscr{G} \rightarrow \mathscr{G}$ - ненулевой вольтерров оператор с $\lambda=0$ в непрерьвном спектре. Тогда диагональньй относительно (3.1) оператор $\widetilde{A}=\operatorname{diag}\left\{A, A^{*}\right\}$ - вольтерров $J$-самосопряженный оператор, $\widetilde{A} \notin \mathbf{K}(\mathbf{H})$ и $\widetilde{A}$ не является дефинизируемьм. В самом деле, если бы $\widetilde{A} \in \mathbf{K}(\mathbf{H})$, то $\widetilde{A}$ имел бы базисную (в смысле Рисса) систему собственных векторов (см. [2, теорема 4.2.12]), а если бы он был дефинизируем, то его система собственных векторов была бы полна (см. [2, лемма 4.2.14]). Оба этих свойства несовместимы с вольтерровостью $\widetilde{A}$. 
ПримеР $3.2\left(\mathbf{D} \not \subset\left(\mathbf{K}(\mathbf{H}) \cup \mathfrak{S}_{\infty, 12}\right)\right)$. Пусть теперь $A: \mathscr{G} \rightarrow \mathscr{G}$ - положительный вполне непрерьвньй оператор. Тогда

$$
\widetilde{A}=\left[\begin{array}{ll}
0 & I \\
A & 0
\end{array}\right]
$$

- $J$-положительньй оператор, имеющий полную систему собственных векторов (см. [2, лемма 4.2.14]), однако в $\mathscr{H}$ не существует базиса, составленного из этих векторов: в противном случае $\widetilde{A} \in \mathfrak{S}_{\infty}$. Следовательно, этот оператор не принадлежит классу $\mathbf{K}(\mathbf{H})$. Более того, (3.3) - пример дефинизируемого (неотрицательного) $J$-самосопряженного оператора, для которого не существует такого разложения (1.1), что относительно него $\widetilde{A}$ имеет матричное представление с вполне непрерьвньм уголком, т.е. $\widetilde{A} \notin \mathfrak{S}_{\infty, 12}$. Последнее следует из равенства $\widetilde{A}=A_{1}+A_{2}$, где

$$
A_{1}=\left[\begin{array}{ll}
0 & 0 \\
A & 0
\end{array}\right], \quad A_{2}=\left[\begin{array}{ll}
0 & I \\
0 & 0
\end{array}\right],
$$

$A_{1} \in \mathfrak{S}_{\infty}, A_{2}-J$-неотрицательный оператор и $A_{2}^{2}=0$. Если бы существовало такое разложение (1.1), что $A$ имело бы вполне непрерьвньй уголок, то $A_{2}$ также имел бы вполне непрерьвньй уголок относительно того же разложения. В этом случае имеем $A_{2}^{2}=0$ и вполне непрерьвность оператора $A_{2}$, что противоречит бесконечномерности $\mathscr{G}$.

ПримеР 3.3 (H $\not \mathbf{D})$. Искомьй пример $\widetilde{A}$ указать достаточно просто: можно рассмотреть относительно (1.1) диагональную блочно-операторную матрицу $\widetilde{A}=\left\{A_{1}, A_{2}\right\}$ с вполне непрерьвньпи самосопряженными циклическими операторами $A_{1}, A_{2}$, собственные значения которых отличны от нуля и перемежаются. Однако несложно представить в этом случае $\mathscr{K}$ как прямую ортогональную сумму таких двух $\widetilde{A}$-инвариантных подпространств Крейна, что сужения оператора $\widetilde{A}$ на эти подпространства - дефинизируемые операторы; более того, эти подпространства можно выбрать как пространства Понтрягина. Таким образом, изучение $\widetilde{A}$ сводится к исследованию дефинизируемых операторов или операторов в пространствах Понтрягина, соответственно.

Мы модифицируем этот пример и укажем такой оператор $\widetilde{A}$, что нельзя провести подобную редукцию к дефинизируемым операторам или, в частности, к операторам в пространстве Понтрягина.

Пусть $\mathscr{E}=Л О\{e\}$ - одномерное пространство и $\mathscr{G}$ - сепарабельное бесконечномерное пространство (ЛО - линейная оболочка). Образуем пространство Крейна $\mathscr{K}$ как следующее $J$-пространство:

$$
\mathscr{K}=\mathscr{E} \oplus \mathscr{G} \oplus \mathscr{G} \oplus \mathscr{E}
$$

с оператором

$$
J=\left[\begin{array}{cccc}
0 & 0 & 0 & 1 \\
0 & I & 0 & 0 \\
0 & 0 & -I & 0 \\
1 & 0 & 0 & 0
\end{array}\right] .
$$

Пусть $\left\{f_{n}\right\}$ и $\left\{g_{n}\right\}$ - ортонормированные базисы в $\mathscr{G}$. Положим

$$
f=\sum_{n=1}^{\infty} \frac{1}{2 n} f_{n} \quad \text { и } \quad g=\sum_{n=1}^{\infty} \frac{1}{2 n-1} g_{n} .
$$


Операторы

$$
A_{22}=\sum_{n=1}^{\infty} \frac{1}{2 n}\left(\cdot, f_{n}\right) f_{n} \quad \text { и } \quad A_{33}=\sum_{n=1}^{\infty} \frac{1}{2 n-1}\left(\cdot, g_{n}\right) g_{n}
$$

являются вполне непрерывными, самосопряженными и $f \notin \operatorname{ran} A_{22}, g \notin \operatorname{ran} A_{33}$. Зададим оператор $\widetilde{A}$ как блочно-операторную $(4 \times 4)$-матрицу относительно $(3.4)$ :

$$
\widetilde{A}=\left[\begin{array}{cccc}
0 & A_{12} & A_{13} & 0 \\
0 & A_{22} & 0 & A_{12}^{*} \\
0 & 0 & A_{33} & -A_{13}^{*} \\
0 & 0 & 0 & 0
\end{array}\right]
$$

где $A_{12}=(\cdot, f) e, A_{13}=(\cdot, g) e$. Определенньй так оператор $\widetilde{A}$ является вполне непрерьвньг, $J$-самосопряженным относительно $J$ из $(3.5)$, принадлежит классу Н и не может быть разложен в конечную сумму дефинизируемых операторов.

ПРИМЕР $3.4\left(\mathbf{D}_{\kappa}^{+} \neq \mathbf{K}(\mathbf{H})\right)$. Пусть $\left\{e_{k}\right\}_{k=-\infty}^{\infty} \cup\left\{g_{0}\right\}-$ ортонормированньй базис в гильбертовом пространстве $\mathscr{K}$. Положим

$$
\begin{aligned}
& J e_{k}=e_{k}, \quad J e_{-k}=-e_{k}, \quad k=1,2, \ldots, \quad J e_{0}=g_{0}, \quad J g_{0}=e_{0}, \\
& A_{k} x=e_{k}\left[x, e_{0}\right]+e_{0}\left[x, e_{k}\right], \quad \text { где } x \in \mathscr{H}, \quad \pm k=1,2, \ldots
\end{aligned}
$$

Семейство операторов $\left\{A_{k}\right\}_{ \pm k=1}^{\infty}$ принадлежит классу $\mathbf{D}_{1}^{+}$, однако, не входит в класс $\mathbf{K}(\mathbf{H})$. Действительно, все операторы из $\left\{A_{k}\right\}_{ \pm k=1}^{\infty}$ являются $J$-самосопряженными, подпространство $\mathscr{L}_{+}=$ЗЛО $\left\{e_{k}\right\}_{k=0}^{\infty}$ (здесь и всюду ниже ЗЛО - замкнутая линейная оболочка) инвариантно по отношению к $\left\{A_{k}\right\}_{ \pm k=1}^{\infty}$ и $\mathscr{L}_{+} \in \mathfrak{M}^{+} \cap \mathbf{h}^{+}$. Кроме того, $\operatorname{dim}\left(\mathscr{L}_{+} \cap \mathscr{L}_{+}^{[\perp]}\right)=1$, так что $\left\{A_{k}\right\}_{ \pm k=1}^{\infty} \in \mathbf{D}_{1}^{+}$. Далее, линейная оболочка, натянутая на вектор $e_{0}$, является общим ядром семейства $\left\{A_{k}\right\}_{ \pm k=1}^{\infty}$. Допустим, что $J$-самосопряженньй оператор $B$ коммутирует с $\left\{A_{k}\right\}_{ \pm k=1}^{\infty}$. Тогда вектор $e_{0}$ собственный для $B$. Без потери общности можно предположить, что $B e_{0}=0$. В этом случае $B g_{0} \in$ ЗЛО $\left\{e_{k}\right\}_{k=-\infty}^{\infty}$. Найдем $B e_{k}$ для $\pm k=1,2, \ldots: B e_{k}=B A_{k} g_{0}=A_{k} B g_{0}=$ $\beta_{k} e_{0}$. В силу последнего мы получаем следующее представление оператора $B: B x=$ $[x, z] e_{0}+\left[x, e_{0}\right] z+\xi\left[x, e_{0}\right] e_{0}$, где $z=\sum_{k=-\infty, k \neq 0}^{\infty} \gamma_{k} e_{k}$. Однако ядро этого оператора имеет бесконечньй ранг индефинитности и поэтому $B \notin \mathbf{H}$.

4. Операторы классов $\mathbf{D}_{\kappa}^{+}$и $\mathbf{K}(\mathbf{H})$. Ниже мы покажем, что для конечного семейства коммутирующих операторов принадлежность классу $\mathbf{D}_{\kappa}^{+}$влечет включение в $\mathbf{K}(\mathbf{H})$.

ТЕОремА 4.1. Пусть $\mathfrak{A}=\left\{A_{j}\right\}_{j=1}^{m}$ - конечное семейство ограниченных попарно коммутируюших $J$-самосопряхенных операторов в пространстве Крейна. Тогда условия $\mathfrak{A} \in \mathbf{D}_{\kappa}^{+} u \mathfrak{A} \in \mathbf{K}(\mathbf{H})$ эквивалентны.

ДокАЗАтЕльство. Утверждение $\mathfrak{A} \in \mathbf{K}(\mathbf{H}) \Longrightarrow \mathfrak{A} \in \mathbf{D}_{\kappa}^{+}$следует из [2, теоремa 3.5.12].

Докажем, что $\mathfrak{A} \in \mathbf{D}_{\kappa}^{+} \Longrightarrow \mathfrak{A} \in \mathbf{K}(\mathbf{H})$. Покажем существование ограниченного $J$-самосопряженного оператора $X$ класса $\mathbf{H}$, коммутирующего с каждым оператором из 
$A_{j}, j=1, \ldots, k$. По определению класса $\mathbf{D}_{\kappa}^{+}$у семейства $\mathfrak{A}$ существуют инвариантные подпространства $\mathscr{L}^{ \pm} \in \mathfrak{M}^{ \pm}$такие, что $\mathscr{L}^{+}[\perp] \mathscr{L}^{-}$и

$$
\mathscr{L}^{ \pm}=\mathscr{L}^{0} \oplus \mathscr{L}_{ \pm}
$$

где $\mathscr{L}^{0}$ - конечномерное нейтральное подпространство, а $\mathscr{L}_{ \pm}-$равномерно дефинитные подпространства. Без ограничения общности будем считать, что $\mathscr{L}_{ \pm} \subset \mathscr{K}^{ \pm}$, где $\mathscr{K}^{ \pm}$- компоненты (1.1). Обозначим через $K$ угловой оператор подпространства $\mathscr{L}^{+}$, т.е. $K: \mathscr{K}^{+} \rightarrow \mathscr{K}^{-},\|K\| \leqslant 1$ и $\mathscr{L}^{+}=\left\{x=x^{+}+K x^{+} \mid x^{+} \in \mathscr{K}^{+}\right\}$. Пусть $A_{j}=\left\|A_{j i k}\right\|_{i, k=1}^{2}-$ матричное представление операторов $A_{j}, j=1, \ldots, m$, относительно (1.1). Будем искать оператор $X$ в виде матрицы

$$
X=\left[\begin{array}{ll}
X_{11} & X_{12} \\
X_{21} & X_{22}
\end{array}\right],
$$

компоненты которой удовлетворяют следующим условиям: $X_{11}=X_{11}^{*}, X_{21}=K X_{11}$, $X_{12}=-X_{21}^{*}=-X_{11} K^{*}, X_{22}=X_{22}^{*}=-K X_{11} K^{*}$. Поскольку $\mathscr{L}_{ \pm} \subset \mathscr{K}^{ \pm}$, оператор $K$ является конечномерным частично изометрическим. Поэтому из представления (4.2) следует, что $X \in \mathbf{H}$ тогда и только тогда, когда $\operatorname{dim} \operatorname{ker} X_{11}<\infty . A_{j}$-инвариантность $\mathscr{L}^{+}$равносильна тому, что

$$
K A_{j 11}+K A_{j 12} K-A_{j 21}-A_{j 22} K=0, \quad j=1, \ldots, m .
$$

Из (4.3) следует, что $X$ коммутирует с $A_{j}$ тогда и только тогда, когда $\left(A_{j 11}+A_{j 12} K\right) X_{11}$ - самосопряженный оператор в $\mathscr{K}^{+}$, что в свою очередь эквивалентно самосопряженности оператора

$$
\left[\left(P^{+} \mid \mathscr{L}^{+}\right)^{-1}\left(A_{j 11}+A_{j 12} K\right)\left(P^{+} \mid \mathscr{L}^{+}\right)\right]\left[\left(P^{+} \mid \mathscr{L}^{+}\right)^{-1} X_{11}\left(P^{+} \mid \mathscr{L}^{+}\right)^{-1 *}\right] .
$$

Поскольку по условию $\mathscr{L}^{+}$инвариантно относительно $A_{j}$ и по построению относительно $X$, то из (4.4) и соотношений

$$
A_{j} \mid \mathscr{L}^{+}=\left(P^{+} \mid \mathscr{L}^{+}\right)^{-1}\left(A_{j 11}+A_{j 12} K\right)\left(P^{+} \mid \mathscr{L}^{+}\right)
$$

следует, что коммутируемость $A_{j}$ и $X$ эквивалентна существованию такого самосопряженного оператора $Y: \mathscr{L}^{+} \rightarrow \mathscr{L}^{+}$с $\operatorname{dim} \operatorname{ker} Y<\infty$ и $Z_{j} Y=Y Z_{j}^{*}$, где $Z_{j}=A_{j} \mid \mathscr{L}^{+}$, $j=1, \ldots, m,\left(Y=\left(P^{+} \mid \mathscr{L}^{+}\right)^{-1} X_{11}\left(P^{+} \mid \mathscr{L}^{+}\right)^{-1 *}\right)$.

Пусть $Z_{j}=\left\|Z_{j i k}\right\|_{i, k=1}^{2}-$ матричное представление оператора $Z_{j}$ относительно разложения (4.1), $j=1, \ldots, m$. Тогда $Z_{j 21}=0$ и $Z_{j 22}$ - самосопряженный оператор. Пусть $\sigma\left(Z_{j 11}\right)=\left\{\lambda_{j s}\right\}_{s=1}^{s_{j}}-$ спектр оператора $Z_{j 11}, P_{0}-$ ортопроектор из $\mathscr{L}_{+}$на $\mathscr{H}_{0}=$ $\cap\left\{Л О\left\{\operatorname{ker}\left(Z_{j 22}-\lambda_{j s}\right)\right\}_{s=1}^{s}\right\}_{j=1}^{m}$ и $\mathscr{M}-$ минимальное подпространство, содержащее ЛО $\left\{P_{0} Z_{j 12}^{*} \mathscr{L}^{0}\right\}_{j=1}^{m}$ и инвариантное относительно $Z_{j 22}, j=1, \ldots, m$. Отсюда $\operatorname{dim} \mathscr{M}<\infty$, подпространство $\mathscr{H}_{0}$ инвариантно относительно всех операторов $Z_{j 22}$ и существует такое разложение

$$
\mathscr{L}_{+}=\mathscr{H}_{0} \oplus \mathscr{H}_{1} \oplus \cdots \oplus \mathscr{H}_{m}, \quad Z_{j 22} \mathscr{H}_{k} \subset \mathscr{H}_{k}, \quad j, k=1, \ldots, m
$$

что

$$
\sigma\left(Z_{j 11} \cap \sigma_{p}\left(Z_{j 22} \mid \mathscr{H}_{j}\right)=\varnothing, \quad j=1, \ldots, m\right.
$$


где символом $\sigma_{p}$ обозначено множество собственных значений соответствующего оператора. Пусть $Y=\left\|Y_{i k}\right\|_{i, k=1}^{2}-$ матричное представление искомого оператора $Y$. Тогда $Z_{j} Y$ - самосопряженньй оператор тогда и только тогда, когда выполнены следующие соотношения:

(i) $Z_{j 11} Y_{11}+Z_{j 12} Y_{12}^{*}=Y_{11} Z_{j 11}^{*}+Y_{12} Z_{j 12}^{*}$;

(ii) $Y_{12}^{*} Z_{j 11}^{*}+Y_{22} Z_{j 12}^{*}=Z_{j 22} Y_{12}^{*}$

(iii) $Z_{j 22}=Y_{22} Z_{j 22}$.

Это дает нам основание строить $Y$ так, чтобы каждое из подпространств разложения (4.5) было инвариантным относительно $Y_{22}$. Положим $Y=\sum_{j=0}^{m} Y_{j}$, где $Y_{j}=$ $\left\|Y_{j i k}\right\|_{i, k=1}^{2}$ удовлетворяют условиям (i)-(iii) и $Y_{j 22} \mid \mathscr{H}_{k}=0$ при $j \neq k, j, k=1, \ldots, m$. Если операторы $Y_{j 22}$ мы выберем так, чтобы $\operatorname{dim} \operatorname{ker} Y_{j 22} \mid \mathscr{H}_{j}<\infty$, то построенньй $Y$ будет искомым оператором.

Определим операторы $Y_{j}, j=0,1, \ldots, m$, следуюшим образом: $Y_{0}: Y_{011}=0, Y_{012}=0$, $Y_{022}$ - ортопроектор в $\mathscr{H}_{0}$ на $\mathscr{M}^{\perp} ; Y_{j}, j=1, \ldots, m$. Пусть $\widetilde{Z}_{j}=\prod_{s=1}^{s_{j}}\left(Z_{j}-\lambda_{j s}\right)\left(Z_{j}-\bar{\lambda}_{j s}\right)$ и $\widetilde{Z}_{j}=\left\|\widetilde{Z}_{j i k}\right\|_{i, k=1}^{2}-$ его матричное представление относительно (4.1). Положим $Y_{j 11}=$ $\widetilde{Z}_{j 12} \widetilde{Z}_{j 12}^{*}, Y_{j 12}=\widetilde{Z}_{j 12} \widetilde{Z}_{j 22}, Y_{j 21}=Y_{j 12}^{*}, Y_{j 22}=\widetilde{Z}_{j 22}, j=1, \ldots, m$.

5. Дефинизируемые операторы и класс $\mathbf{K}(\mathbf{H})$. Дефинизируемые операторы и операторы класса $\mathbf{K}(\mathbf{H})$ имеют ряд похожих свойств. В частности, если $J$-самосопряженньй оператор принадлежит хотя бы одному из этих двух классов, то он обладает спектральной функцией с конечным множеством спектральных особенностей. Характер этих особенностей (в случае дефинизируемого оператора они входят в, вообще говоря, более широкое множество критических точек) для двух указанных случаев различен, поэтому естественен вопрос о спектральньх особенностях оператора, входящего одновременно в оба класса.

Ниже в формулировке теоремы 5.3 и ее доказательстве мы будем использовать общепринятую терминологию, касающуюся $J$-спектральной функции дефинизируемого оператора и ее критических точек [7] (см. также [2, гл. IV]).

Существование спектральной функции у оператора класса $\mathbf{K}(\mathbf{H})$ в виде упражнения было анонсировано впервые в [2]. Приведем соответствующий результат в той форме, как он подробно доказан в [8].

ПРЕДЛОЖЕНИЕ 5.1. Пусть спектр J-самосопряжсенного оператора $A \in \mathbf{D}_{\kappa}^{+}$вещественен, $\mathscr{L}_{+}$- отвечающее определению 2.5 подпространство, инвариантное относительно $A$. Тогда оператор $A$ обладает спектральной функиией $E_{\lambda} c$ конечным множеством спектральных особенностей $\Lambda \subset \mathbb{R}$, причем

а) $E_{\lambda} \in \operatorname{Alg} A$ для любого $\lambda \in \mathbb{R} \backslash \Lambda, \sigma\left(\left.A\right|_{E(\Delta)} \mathscr{K}\right) \subset \bar{\Delta}$ для любого $\Delta \in \mathscr{R}_{\Lambda}$, $E(\mathbb{R})=I$

b) если $\Delta \in \mathscr{R}_{\Lambda} u \Delta \cap \Lambda=\varnothing$, mo $E(\Delta) \mathscr{K}=\mathscr{K}_{\Delta}^{+}[+] \mathscr{K}_{\Delta}^{-}, A \mathscr{K}_{\Delta}^{+} \subset \mathscr{K}_{\Delta}^{+}$, $A \mathscr{K}_{\Delta}^{-} \subset \mathscr{K}_{\Delta}^{-}, \mathscr{K}_{\Delta}^{+}=E(\Delta) \mathscr{L}_{+}-$равномерно положительное, а $\mathscr{K}_{\Delta}^{-}-$равномерно отричательное подпространство (каждое из подпространств $\mathscr{K}_{\Delta}^{+}$ и $\mathscr{K}_{\Delta}^{-}$мохсет вырождаться в $\left.\{0\}\right) ;$

c) если $\Delta \in \mathscr{R}_{\Lambda} u \Delta \cap \Lambda \neq \varnothing$, mo $E(\Delta)\left(\mathscr{L}_{+} \cap \mathscr{L}_{+}^{[\perp]}\right) \neq\{0\}$

где $\operatorname{Alg} A$ - минимальная слабо замкнутая алгебра, порожденная $A$ и содержащая единииу, $\mathscr{R}_{\Lambda}$ - система всех таких борелевских подмнохсеств вещественной 
оси, для которых никакая точка из $\Lambda$ не является точкой прикосновения, хотя и мохет быть внутренней точкой.

ЗАмечание 5.2. Если $J$-самосопряженньй оператор $A \in \mathbf{K}(\mathbf{H})$ имеет невещественньй спектр, то он также обладает спектральной функцией, определенной на $\mathbb{R} \backslash \Lambda$, однако в этом случае $E(\mathbb{R}) \neq I$ и $\sigma\left(\left.A\right|_{(I-E(\mathbb{R}))} \mathscr{K}\right) \subset \mathbb{C} \backslash \mathbb{R}$.

Перейдем теперь к результатам настоящего раздела.

Терема 5.3. Для того чтобы J-самосопряжсенный оператор $A \in \mathbf{K}(\mathbf{H}) \quad c$ инвариантными подпространствами $\mathscr{L}^{ \pm} \in \mathfrak{M}^{ \pm}, \mathscr{L}^{+}[\perp] \mathscr{L}^{-}$был дефинизируемым, необходимо и достаточно, чтобы существовало конечное множсество точек $\left\{\mu_{k}\right\}_{k=1}^{m} \subset \mathbb{R}$ таких, что интерваль $\left(-\infty, \mu_{1}\right),\left(\mu_{1}, \mu_{2}\right), \ldots,\left(\mu_{m}, \infty\right)$ состоят из точек дефинитного типа J-спектральной функиии E оператора $A$.

ДокАЗАТЕЛЬСТВО. Необходимость следует из общей теории дефинизируемьх операторов [7].

Достаточность. Если $J$-самосопряженньй оператор $A \in \mathbf{K}(\mathbf{H})$ имеет невещественный спектр, то последний состоит из конечного числа конечнократных собственных значений $\left[2\right.$, следствие 3.5.21]. Пусть $\left\{\lambda_{j}, \bar{\lambda}_{j}\right\}_{j=1}^{s}-$ множество невещественньх собственньх значений оператора $A$, если они сушествуют. Введем обозначения:

$$
\mathscr{K}_{1}=\sum_{j=1}^{s} \mathscr{L}_{\lambda_{j}}(A)+\mathscr{L}_{\bar{\lambda}_{j}}(A)
$$

где $\mathscr{L}_{\lambda_{j}}(A)$ и $\mathscr{L}_{\bar{\lambda}_{j}}(A)$ - корневые линеалы, отвечающие $\lambda_{j}$ и $\bar{\lambda}_{j}$ соответственно, $j=1$, $\ldots, s, \mathscr{K}_{2}=\mathscr{K}_{1}^{[\perp]}$. Тогда $\mathscr{K}_{1}$ и $\mathscr{K}_{2}$ инвариантны относительно $A$ и $\mathscr{K}=\mathscr{K}_{1}[\dot{+}] \mathscr{K}_{2}$. Обозначим $A_{j}=A \mid \mathscr{K}_{j}, j=1,2$. Оператор $A_{2}$ обладает теми же свойствами, что и $A$, и дополнительным свойством, что его спектр вещественен. Отсюда следует существование такого многочлена $p$ с множеством нулей, содержашим $\left\{\lambda_{j}, \bar{\lambda}_{j}\right\}_{j=1}^{s},\left\{\mu_{k}\right\}_{k=1}^{m}$, что оператор $B:=p(A)$ обладает тем свойством, что отрицательная полуось состоит из точек отрищательного типа, а положительная - из точек положительного типа. Поскольку у оператора $A$ длины жордановых цепочек равномерно ограничены [2, следствие 3.5.21], то $p$ выберем так, чтобы $\mathscr{L}_{0}(B)=\operatorname{ker} B$. По условию подпространства $\mathscr{L}^{ \pm}$инвариантны относительно $A$, а потому и относительно $B$. Пусть $\mathscr{L}^{0}=\mathscr{L}^{+} \cap \mathscr{L}^{-}$. Тогда

$$
\mathscr{K}=\mathscr{L}^{0} \dot{+} \mathscr{L}_{+} \dot{+} \mathscr{L}_{-} \dot{+} J \mathscr{L}^{0},
$$

где $\mathscr{L}_{ \pm}-$равномерно дефинитные компоненты разложения подпространств $\mathscr{L}^{+}$. Относительно (5.1) оператор $B$ представляется матрицей

$$
B=\left[\begin{array}{cccc}
0 & B_{12} & B_{13} & B_{14} \\
0 & B_{22} & 0 & B_{24} \\
0 & 0 & B_{33} & B_{34} \\
0 & 0 & 0 & 0
\end{array}\right]
$$

Так как $\mathscr{L}_{+}-$равномерно положительное подпространство, а $\mathscr{L}_{-}-$равномерно отрицательное, то $\sigma\left(B_{22}\right) \in[0, \infty)$, а $\sigma\left(B_{33}\right) \in(-\infty, 0]$. Отсюда следует, что оператор $B$ 
является конечномерным возмущением $J$-неотрицательного оператора

$$
B_{1}=\left[\begin{array}{cccc}
0 & 0 & 0 & 0 \\
0 & B_{22} & 0 & 0 \\
0 & 0 & B_{33} & 0 \\
0 & 0 & 0 & 0
\end{array}\right] .
$$

В силу [9] оператор $B$, а с ним и оператор $A$, являются дефинизируемыми.

Перейдем теперь к вопросу о том, чем отличается спектральная функция оператора класса $\mathbf{H}$ от спектральной функции оператора класса $\mathbf{D}_{\kappa}^{+}$. Отметим, прежде всего, следующее предложение.

ПРЕДЛОЖЕНИЕ 5.4. Пусть $A \in \mathbf{H}-J$-самосопряженный оператор. Тогда всем его вещественным собственным числам, за исключением, быть может, их конечного числа, отвечают равномерно дефинитные собственные подпространства, $а$ собственные подпространства, отвечающие исключительным собственным значениям, регулярны или псевдорегулярны, имеют конечный ранг индефинитности и конечномерную изотропную часть.

ЗАмечАниЕ 5.5. Пусть $E_{\lambda}$ - спектральная функция $J$-самосопряженного оператоpa $A \in \mathbf{D}_{\kappa}^{+}$. Функцию $f(\lambda)$, заданную на множестве $\operatorname{Supp}(E) \backslash \Lambda(\operatorname{Supp}-$ носитель меры), назовем $E$-измеримой, если она измерима по отношению к любой мере Лебега-Стильтьеса $\mu_{\sigma}$, задаваемой функцией вида $\sigma(\lambda)=\left[E_{\lambda} x, x\right]$,

$$
x \in \widetilde{\mathscr{K}}=\underset{\Delta \in \mathscr{R}_{\Lambda}, \Delta \cap \Lambda=\varnothing}{3 \mathrm{C}_{\Lambda}} E(\Delta) \mathscr{K},
$$

определенной первоначально на $\operatorname{Supp}(E) \backslash \Lambda$ и доопределенной по непрерывности в точках из $\Lambda$. Если пространство $\mathscr{K}$ сепарабельно, то измеримость функции по отношению к указанной совокупности мер Лебега-Стильтьеса может быть заменена измеримостью по отношению к единственной мере $\mu_{\sigma}$, задаваемой функцией $\sigma(\lambda)=\left[E_{\lambda} x, y\right]$, определяемой некоторыми специально подобранньги векторами $x$ и $y$.

ДокАЗАтЕльство. Действительно, $J$-самосопряженньй оператор $A \in \mathbf{D}_{\kappa}^{+}$порождает в фактор-пространстве $\widehat{\mathscr{K}}=\widetilde{\mathscr{K}} /\left(\widetilde{\mathscr{K}} \cap \widetilde{\mathscr{K}}^{[\perp]}\right)$ оператор $\widehat{A}$, самосопряженный по отношению к подходящим образом выбранному каноническому скалярному произведению, причем $\sigma_{p}(A) \cap \Lambda=\varnothing$. По поводу дальнейшего см., например, [10, п. 76, теорема 1]. Для наших целей целесообразно выбрать указанные вектора специальным образом. Положим

$$
\widetilde{\mathscr{K}}_{+}=\underset{\Delta \in \mathscr{R}_{\Lambda}, \Delta \cap \Lambda=\varnothing}{3 J_{0}}\left\{\mathscr{K}_{+}(\Delta)\right\}, \quad \widetilde{\mathscr{K}}_{-}=\underset{\Delta \in \mathscr{R}_{\Lambda}, \Delta \cap \Lambda=\varnothing}{3 \pi_{\Lambda} O}\left\{\mathscr{K}_{-}(\Delta)\right\},
$$

где $\mathscr{K}_{+}(\Delta)$ и $\mathscr{K}_{-}(\Delta)$ взяты в соответствии с условиями а)-c) предложения 5.1. Тогда найдутся такой вектор $x_{+} \in \widetilde{\mathscr{K}}_{+}$, что измеримость множества $X \subset \mathbb{R} \backslash \Lambda$ по отношению к мере $\mu_{\sigma_{+}}$, где $\sigma_{+}(\lambda)=\left[E_{\lambda} x_{+}, x_{+}\right]$, влечет измеримость $X$ по отношению к мере $\mu_{\sigma}$, где $\sigma(\lambda)=\left[E_{\lambda} y, y\right]$, для любого $y \in \widetilde{\mathscr{K}}_{+}$, и аналогичный вектор $x_{-} \in \widetilde{\mathscr{K}}_{-}$. Искомые вектора $x$ и $y$, которые исчерпывающим образом описьвают $E_{\lambda}$-измеримость, возьмем теперь в виде

$$
x=x_{+}+x_{-}, \quad y=x_{+}-x_{-} .
$$

Из примера 3.3 вытекает, что операторы класса $\mathbf{H}$, вообще говоря, не дефинизируемы, однако приводимая ниже теорема показьвает, что в некотором отношении операторы класса Н похожи на дефинизируемые операторы. 
Теорема 5.6. Пусть пространство $\mathscr{K}$ сепарабельно, $A \in \mathbf{H}-J$-самосопряженный оператор, $E_{\lambda}$ - его спектральная функиия с множеством критических точек $\Lambda$. Тогда найдется такое конечное множество $\widehat{\Lambda} \subset \mathbb{R}, \Lambda \subset \widehat{\Lambda}, u E_{\lambda}$-измеримые множества $X_{+}, X_{-} \subset \mathbb{R}, X_{+} \cap X_{-}=\varnothing, X_{+} \cup X_{-}=\mathbb{R} \backslash \widehat{\Lambda}$, что для любого отрезка $\Delta \in B_{\Lambda} \cap \mathbb{R}$ каждое из подпространств

$$
E\left(\Delta \cap X_{+}\right) \mathscr{K} \quad u \quad E\left(\Delta \cap X_{-}\right) \mathscr{K}
$$

либо вырождается в $\{0\}$, либо, соответственно, положительно и отрицательHO.

ДокАЗАТЕЛЬСтво. Образуем множество $\widehat{\Lambda}$, присоединив к $\Lambda$ те $\lambda \in \sigma_{p}(A)$, для которых соответствующее собственное подпространство индефинитно. В силу предложения $5.4 \widehat{\Lambda}$ - конечное множество. Для упрощения выкладок допустим, что $\Lambda=\widehat{\Lambda}$ и при сделанном предположении покажем, что меры, порождаемые функциями $\sigma_{+}(\lambda)=$ $\left[E_{\lambda} x_{+}, x_{+}\right]$и $\sigma_{-}(\lambda)=-\left[E_{\lambda} x_{-}, x_{-}\right]\left(x_{+}\right.$и $x_{-}$те же, что и в $\left.(5.4)\right)$, взаимно сингулярны. Отметим, во-первых, что в силу сделанного допущения взаимно сингулярны атомические компоненты этих мер, поэтому можно считать, что функции $\sigma_{+}(\lambda)$ и $\sigma_{-}(\lambda)$ непрерьвны.

В соответствии с замечанием 5.5 и разложением (5.4) имеем $\sigma(\lambda)=\sigma_{+}(\lambda)+\sigma_{-}(\lambda)$, откуда $\mu_{\sigma_{+}} \prec \mu_{\sigma}$ и $\mu_{\sigma_{-}} \prec \mu_{\sigma}(\prec-$ символ подчиненности мер), поэтому

$$
\sigma_{+}(\lambda)=\int_{-\infty}^{\lambda} \rho_{+}(\tau) d \sigma(\tau), \quad \sigma_{-}(\lambda)=\int_{-\infty}^{\lambda} \rho_{-}(\tau) d \sigma(\tau)
$$

причем для любого $\tau \in \mathbb{R}$ справедливо $\rho_{+}(\tau), \rho_{-}(\tau) \in[0 ; 1]$. Положим $Y=\left\{\tau: \rho_{+}(\tau) \neq 0\right.$, $\left.\rho_{-}(\tau) \neq 0\right\}$. Утверждение теоремы будет доказано, если $\mu_{\sigma} Y=0$. Допустим противное, т.е. что $\mu_{\sigma} Y \neq 0$, и введем две функции:

$$
\begin{aligned}
& f_{+}(t)= \begin{cases}\left(\frac{\rho_{+}(\tau)+\rho_{-}(\tau)}{\rho_{+}(\tau)}\right)^{1 / 2} & \text { при } t \in Y, \\
0 & \text { при } t \notin Y,\end{cases} \\
& f_{-}(t)= \begin{cases}\left(\frac{\rho_{+}(\tau)+\rho_{-}(\tau)}{\rho_{-}(\tau)}\right)^{1 / 2} & \text { при } t \in Y, \\
0 & \text { при } t \notin Y .\end{cases}
\end{aligned}
$$

Зафиксируем теперь какой-либо промежуток $\Delta \subset \mathbb{R}, \Delta \in B_{\Lambda}$, для которого $\mu_{\sigma}(Y \cap \Delta) \neq 0$, и положим

$$
y_{+}=\int_{\Delta} f_{+}(t) d E_{t} x_{+}, \quad y_{-}=\int_{\Delta} f_{-}(t) d E_{t} x_{-} .
$$

Пусть $\Delta_{1} \subset \Delta, z_{\Delta_{1}}=E\left(\Delta_{1}\right)\left(y_{+}+y_{-}\right)$. Тогда

$$
\begin{aligned}
{\left[z_{\Delta_{1}}, z_{\Delta_{1}}\right] } & =\int_{\Delta_{1}} f_{+}^{2}(t) d \sigma_{+}(t)-\int_{\Delta_{1}} f_{-}^{2}(t) d \sigma_{-}(t) \\
& =\int_{\Delta_{1} \cap Y}\left(\rho_{+}(t)+\rho_{-}(t)\right) d \sigma(t)-\int_{\Delta_{1} \cap Y}\left(\rho_{+}(t)+\rho_{-}(t)\right) d \sigma(t)=0 .
\end{aligned}
$$


Положим $\mathscr{L}=3 Л \mathrm{\Delta}_{\Delta \Delta_{1}}\left\{z_{\Delta_{1}}\right\}$. Ясно, что $\mathscr{L}$ - нейтральное подпространство, а поскольку $\mu_{\sigma}(Y \cap \Delta) \neq 0$ и множество $Y \cap \Delta$ свободно от атомической меры, то $\mathscr{L}$ - бесконечномерное подпространство. Итак, оператор $A$ имеет бесконечномерное нейтральное инвариантное подпространство, что невозможно по определению класса $\mathbf{H}$.

В заключение приносим свою благодарность П. Йонасу (Технический университет г. Берлин), неоднократно поднимавшему вопрос о связи дефинизируемых операторов и операторов классов $\mathbf{H}$ и $\mathbf{K}(\mathbf{H})$, что в конечном счете и стимулировало настоящее исследование.

\section{СПИСОК ЦИТИРОВАННОЙ ЛИТЕРАТУРЫ}

[1] Азизов Т.Я., Иохвидов И.С. Линейные операторы в пространствах с индефинитной метрикой и их приложения // Итоги науки и техники. Матем. анализ. Т. 17. М.: ВИНИТИ, 1979. С. $113-205$.

[2] Азизов Т.Я., Иохвидов И.С. Основы теории линейных операторов в пространствах с индефинитной метрикой. М.: Наука, 1986.

[3] Bognar J. Indefinite Inner Product Spaces. Berlin: Springer-Verlag, 1974.

[4] Сухочева Л.И. О свойствах операторов класса Helton'a // Вестн. Воронежского гос. ун-та. Сер. физ., матем. 2001. №1. С. 120-123.

[5] Штраус В.А. О структуре операторов дважды перестановочных с оператором класса $\mathbf{K}(\mathbf{H}) / /$ Укр. матем. ж. 1986. Т. 38. №6. С. 805.

[6] Штраус В.А. О структуре семейства коммутирующих $J$-самосопряженных операторов // Укр. матем. ж. 1989. Т. 41. № 10. С. 1431-1433.

[7] Langer H. Spectral functions of definitizable operators in Kreĭn spaces // Lect. Notes in Math. V. 948. Berlin-Heidelberg-New York: Springer-Verlag, 1982. P. 1-46.

[8] Azizov T. Ya., Strauss V. A. Spectral decompositions for classes of self-adjoint and normal operators on Krein spaces // Spectral Theory and its Applications. Proceedings. Theta, 2003. P. $45-67$.

[9] Jonas P., Langer H. Compact perturbations of definitizable operators // J. Operator Theory. 1979. V. 2. P. 63-77.

[10] Ахиезер Н. И., Глазман И. М. Теория линейных операторов в гильбертовом пространстве. Т. 1. Харьков: Вища школа, 1977.

(Т.Я. Азизов, Л. И. Сухочева) Воронежский государственный университет (В. А. Штраус) Университет им. С. Боливара, Венесуэла E-mail : azizov@tom.vsu.ru 